\title{
AN ORIGINAL ACCURATE THEORETICAL AND NUMERICAL APPROACH TO REDEFINE A WIDE CLASS OF DIGITAL SIGNALS*
}

\author{
ROBINSON GAUDINO CAPUTO ${ }^{\dagger}$ and GUSTAVO VARELLA FIGUEIREDO* \\ Pulse Perfect Science and Technology, \\ Wellington Court 2018, 60314, Geneva, IL, USA \\ †robinsoncaputo@me.com \\ † gustavo@pulse-perfect.com \\ MAURICIO SILVEIRA ${ }^{\text {, }}$ \\ Center of Engineering, Modeling and Applied Social Sciences, \\ Federal University of $A B C$, \\ Avenida dos Estados 5001, Village: Bangu, 09210-580, \\ Santo André, SP, Brazil \\ maur.silveira@gmail.com
}

Received 23 October 2012

Accepted 4 September 2013

Published 6 December 2013

\begin{abstract}
The theoretical and numerical analysis proposed in this paper is totally original and the essential purpose is to introduce an innovative procedure to represent some of the more important digital communications signals by using a simple mathematical tool. All new analytical concepts presented here can be used as definitions of these signals and the great improvement is the fact that we can manipulate all of them through some flexible as well as original mathematical operations. This theoretical technique permits us to represent all classic analogical waves by using periodic and non-periodic continuous functions such that they can be seen as a video color signal or a simple sequence of zeros and ones. By using a nonlinear operator, it is possible to define analytically a wide class of important electromagnetic waves.
\end{abstract}

Keywords: Electromagnetic waves; digital communications; numerical approximations; rise time modulation; reciprocal of the Fourier series theorem; mask for digital communications links.

*This paper was recommended by Regional Editor Piero Malcovati.

$\S$ Corresponding author.

The author will develop some professional activities also at the Center of Mathematics, Computation, and Cognition, Federal University of ABC, SP, Brazil, with the academic process under final analysis of transition. 


\section{Introduction}

Over the years, the more important electromagnetic waves were presented in the literature by using classic standards definitions, as we can see in those areas that interface in the field of research of digital communication. ${ }^{1,2}$ Some of these waves have been using also too as blocks of closed mesh for the architecture in many modern control system. ${ }^{3}$

All of these references have standards ways to present the definitions of these classic waves and in many cases it is not possible to detect the subset of smooth functions involved in the numerical process of convergence that permits us to generate any digital as well as analog signal.

One of the more important concepts to be emphasize is the fact that any wave signal is different from the corresponding wave function, just due to the fact that the transition " 0 " to " 1 " or " 1 " to " 0 " give to us a perfect comprehension which type of waves we would like to plot. Generically, from a mathematical point of view, the numerical process of convergence requires that we define a countable set of smooth functions that converges for a discontinuous function. In most cases will be a digital signal, independent not only of the type of digital transition we would like to get, but also as well as the specified bandwidth that must be reached for the limit condition. $^{4}$

Nowadays, different approaches have been attempted to introduce new computational codes with the implementation of an electronic architecture that can serve in the process of synchronization in order to preserve the security of the communication systems. ${ }^{5}$ We can also see in the present literature the presence of new operators for the implementation of the modern FPGA of high speed architectures for image segmentation. ${ }^{6}$

The theoretical analysis presented here can be used for a wide class of digital signal and it is possible to present the numerical process of convergence that involves the modeling of each particular waveform that needs to be analyzed. All analytical concepts are totally original, and some initial results were previously presented by the authors of Refs. 7-9.

By using this analytical approach it is possible to establish some relationship between a sequence of bits and an equivalent analytical polynomial equation. As a direct consequence, this technique permits us to define a linear transform that reduces a train pulse in a classical polynomial function and also does reciprocally. ${ }^{10,11,19}$

These simple ideas discussed initially, are fundamental stakes in the construction of this new analytical approach. The numerical process of convergence adopted in this paper which is being introduced at this point in the current literature, involves an innovative technological approach which the authors define as the DNAx operator. ${ }^{7-11}$ A continuous process of finding new applications for the technique introduced here is part of our work in progress. ${ }^{12-17}$ 


\section{New Theoretical Approach for Modeling the Electromagnetic Waves}

We start by assuming that $p_{n}(x)$ is a real polynomial defined in the standard form:

$$
p_{n}(x)=\sum_{j=0}^{n}\left(a_{j}\right) \cdot\left(x^{j}\right), \quad n \in\{1,2, \ldots, \infty\} .
$$

In our first analysis, we will assume the function $p_{n}(x)$ has only even exponents, which implies we can obtain the following choice of coefficients:

$$
a_{0}=1, \quad n \rightarrow 2 n, \quad a_{j}=0, \quad \forall j \notin\{1, n-1\}, \quad a_{n}=a^{2 n} .
$$

Thus, Eq. (1) can be reduced in the form:

$$
p_{n}(x)=1+(a x)^{2 n}, \quad n \in N=\{1,2, \ldots, \infty\}, \quad x \in R .
$$

For a fixed value of the parameter $a$, we can obtain distinct polynomial configurations with the increasing of the exponent $n$. It will also be easy to detect more variations of the polynomial close to their roots. In addition, there are three fixed points which belong to all graphics of the polynomial for all values of the parameter $n$. An important case must be considered when $a=1$, and we look for the function defined in the limit by:

$$
p(x)=\operatorname{Lim}_{n \rightarrow \infty}\left[p_{n}(x)\right] .
$$

The 2D graphic of the function $p(x)$ is similar to an opened rectangular box centering at the origin without its top line, for $-1 \leq x \leq 1$.

The same analysis can be performed for the case:

$$
q_{n}(x)=1-(a x)^{2 n}, \quad n \in\{1,2, \ldots, \infty\} .
$$

We can carry on the same analysis for $-1 \leq x \leq 1, a=1$, and for the limit function $q(x)$ defined by:

$$
q(x)=\operatorname{Lim}_{n \rightarrow \infty}\left[q_{n}(x)\right]
$$

\section{Basic Format of the $D N A x$ Operator}

After the brief introduction involving the analytical expressions (3) and (5), the first formulation for the $D N A x$ operator can be defined by the function $d_{n}(x)$ through the quotient of the two previous Eqs. (3) and (5), in the form:

$$
d_{n}(x)=\frac{q_{n}(x)}{p_{n}(x)}=\frac{1-(a x)^{2 \cdot n}}{1+(a x)^{2 \cdot n}}, \quad n \in N=\{1,2, \ldots, \infty\}, \quad x \in R .
$$

The same previous analysis in the limit condition permits us to define the operator $D N A x$ mathematically with the first analytical formulation in the form:

$$
d(x)=\operatorname{Lim}_{n \rightarrow \infty}\left[d_{n}(x)\right] .
$$




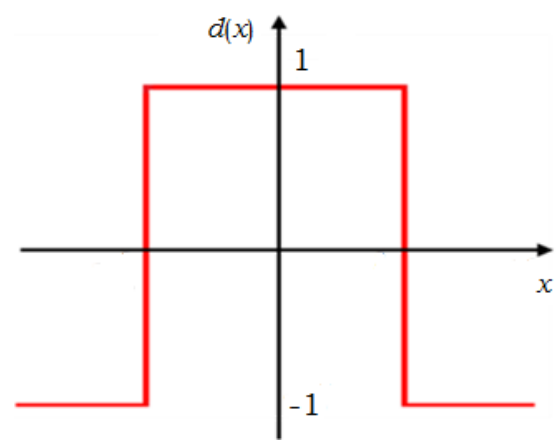

Fig. 1. The rectangular continuous pulse centered at the origin generated with the DNAx operator.

In this case, we will obtain the $2 \mathrm{D}$ graphic of a rectangular continuous pulse centered at the origin. That one localized at the left side has the center at $P_{1}=(-1 / a, 0)$, while the right one has the center at $P_{2}=(1 / a, 0)$ and both up and down transitions go to zero, when $n \rightarrow \infty$. For $a=1$, we obtain the rectangular signal defined by: $d(x)=-1$, for $x<-1$ or $x>1$, while $d(x)=1$, for $-1 \leq x \leq 1$, the vertical lines at the transitions $x \pm 1$, as is depicted in Fig. 1.

At this time, it is important to perform an accurate analysis to check the influence of both real parameters $n$ and $a$, over the width of the window which is known in the present literature as the operational window aperture (OWA). In addition, we can define the Rise Time close to the point $P_{1}$ and confirms that the angle of the straight line with respect to the real axis $x$ tends to $\pi / 2$ when $n \rightarrow \infty$. With similar theoretical arguments we can perform the analysis for the Down Time close to the point $P_{2}=(1 / a, 0)$, which can permit us to visualize the complete transition of the signal from the up to the down levels.

\section{Some Generalization of the $D N A x$ Operator}

\subsection{A more generic representation of the DNAx pulses}

Using the previous definitions, we can make some generalizations, since it is possible to define the rate $y_{n}(x)$ in the form:

$$
y_{n}(x)=\frac{f(x)-(a x)^{2 n}}{1+(a x)^{2 n}}, \quad n \in N=\{1,2, \ldots, \infty\}, x \in R .
$$

In this case, the analytical approach defined by (9) permits us to expose part of the graphic for any arbitrary function $f(x)$ inside some specified window with aperture $\omega$, as is shown in Fig. 2. Small values of the parameter $n$ have direct influence on the up to the down transitions located in both left and right sides of the window. As a consequence, we will have some distortions on $f(x)$ close to both points $P_{1}$ and $P_{2}$. 


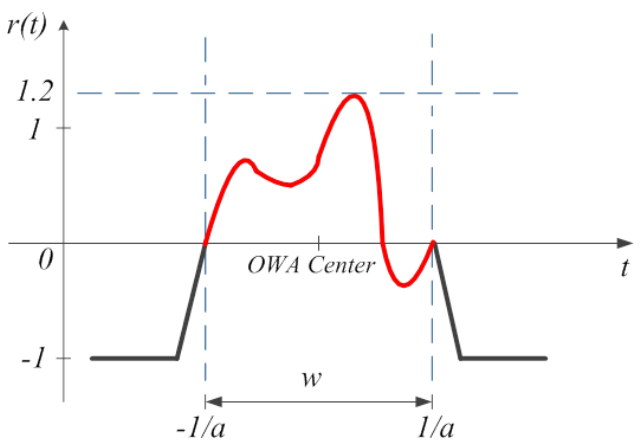

Fig. 2. Generation of an arbitrary function $f(x)$ defined over a window with aperture $\omega$.

\subsection{A large class of the DNAx operator}

If we make a change of the real variable $x$ as equal to the real time $t$, a set of more generalized analytical definitions of the $D N A x$ operator can be formulated. Although we have had some success finding different types of modeling in the current research of digital communication, we will be looking at two new ones, which will be referenced in our future applications.

In that sense, another group of numerical approximations permits us to model a very important class of electromagnetic waves for which the analytical expressions can be defined for the more generic cases in the forms:

$$
\begin{aligned}
& y_{n}(t)=\left[(a \cdot t)^{2 n}+1\right]^{\left[1-(a \cdot t)^{2 n}\right]}, \quad n \in N=\{1,2, \ldots, \infty\}, t \in R, \\
& y_{n}(t)=\left[(a \cdot t)^{2 n}+f(t)\right]^{\left[\left(1-(a \cdot t)^{2 n}\right)\right]}, \quad n \in N=\{1,2, \ldots, \infty\}, t \in R .
\end{aligned}
$$

\section{Original Generation of a Group of Classic Electromagnetic Waves}

As a direct application of these theoretical approaches, by adopting the convergence process defined with the DNAx operator, we will dedicate the final section of this paper to presenting new definitions of some important discontinuous waveforms. Although other analog signals as well as digital ones can be modeled with this operator, in this paper we will focus our analysis only on three important digital signals: the Digital Step Signal, the Digital Rectangular Signal and the Delta of Dirac Signal. ${ }^{18}$

\subsection{Digital step signal}

Using this operator, the Digital Step Signal can be defined in the following form:

$$
\begin{gathered}
u_{n}(t)=[1+a(t)]^{[1-a(t)]}, \quad a(t)=e^{-n \cdot(t-d)}, \\
n \in N=\{1,2, \ldots, \infty\}, \quad t \in R, \quad a \in N,
\end{gathered}
$$


where $N$ and $R$ are the set of the positive integer and the real numbers, respectively; $a$ and $d$ are positive real parameters; and $n$ is a positive integer. There can be an infinite number of choices over these parameters such that the numerical process of convergence can generate the 2D graphic of the Digital Step Signal. This digital communication signal will be defined using our theoretical approach in the limit condition, with the analytic representation:

$$
u(t)=\operatorname{Lim}_{n \rightarrow \infty}\left[u_{n}(t)\right] .
$$

By taking into account that the digital step signal $u(t)$ is obtained as the limit of a family of countable functions $u_{n}(t)$, in order to plot the $2 \mathrm{D}$ graphic for this case, it is enough to choose a fixed value for both real parameters $a$ and $d$, as well as a greater value for the integer parameter $n$. For instance, in the expression (12), we can adopt the following choice of the parameters: $a=3, d=0$ and $n=3.000$.

Figure 3 depicts the $2 \mathrm{D}$ graphic of the function $u_{n}(t)$ with the previous choice of all parameters $a, d$ and $n$, which represents classic Digital Step Signal $u(t)$ defined by Eq. (14). Therefore, we have an analytic theoretical formulation of this important digital signal as a particular application of the $D N A x$ operator.

\subsection{Digital rectangular signal}

In this case, we need to adopt a different process of convergence, and the theoretical approach of this important digital waveform can be expressed as a particular case of the Eq. (10) through the definition:

$$
r_{n}(t)=\left\{\left[1+(a \cdot t)^{2 n}\right]\right\}^{\left\{\left[1-(a \cdot t)^{2 n}\right]\right\}}, \quad n \in N=\{1,2, \ldots, \infty\}, \quad t \in R, a \in N .
$$

With the same argument we mentioned in our first modeling, the Digital Rectangular Signal can be expressed in the limit condition with the analytic theoretical definition:

$$
r(t)=\operatorname{Lim}_{n \rightarrow \infty}\left[r_{n}(t)\right]
$$

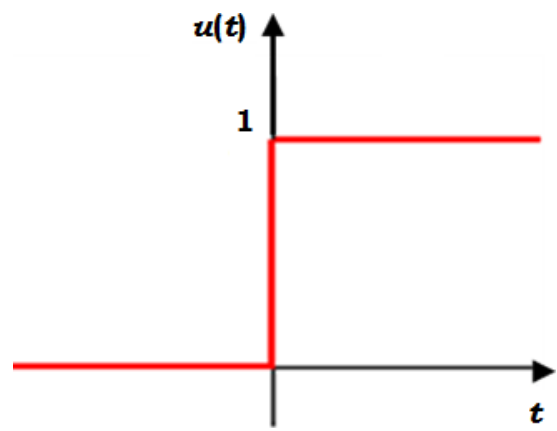

Fig. 3. The Digital Step Signal waveform with the DNAx approach. 


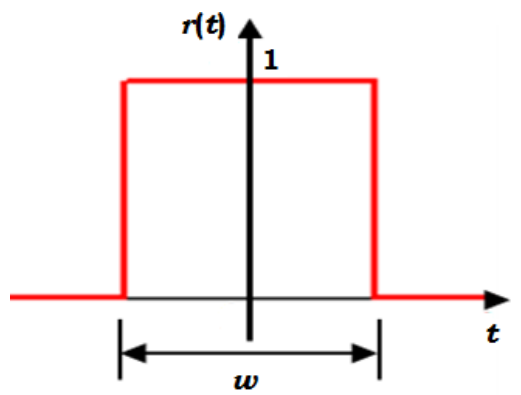

Fig. 4. The Digital Rectangular waveform with the DNAx approach.

For this case, we assume the same choice of parameters used in the previous case; that is $a=3, d=0$ and $n=3.000$. The analysis in the limit condition will derive the classic Digital Rectangular Window $r(t)$, which is shown in the 2D graphic when $n \rightarrow \infty$ as depicted in Fig. 4 .

Finally, we can detect a slight difference between the rectangular continuous pulse centered at the origin and the digital rectangular pulse defined above. Also in this case, it is possible to obtain this important digital signal as a particular case of the $D N A x$ operator.

\subsection{The Delta of Dirac Signal}

Also in this case, another numerical process of convergence must be adopted; and we can define the digital Delta Signal as a real discontinuous function $\delta: R \rightarrow R$ such that by using the countable set of real functions, it can be defined in the form:

$$
\begin{gathered}
\delta_{n}(t)=[1+g(t)]^{[1+g(t)]}, \quad g(t)=(a t)^{-n}, \\
n \in N=\{1,2, \ldots, \infty\}, \quad t \in R, \quad a \in N,
\end{gathered}
$$

where $a$ and $n$ are positive numbers. In this case, since the exponent of the basis in the sequence of functions $\delta_{n}(t)$ and $n \in N$ has a negative signal. In the case that the parameter $a \gg 1$, we will have a negligible value of the bandwidth of the limit function.

Therefore, for this case, to get a good approximation of the Delta Pulse, it is enough to make the following choice of the integer real parameters: $a=10^{12}$ and $n=1000 \ll a$. Also in this case, we can generate this important electromagnetic pulse defined in the limit condition; through the analytic formulation by:

$$
\delta(t)=\operatorname{Lim}_{n \rightarrow \infty}\left[\delta_{n}(t)\right]
$$

The Dirac Signal defined as in Eq. (18) has the 2D graphic depicted in Fig. 5, and in this case it is possible to detect that we have a waveform with temporal aperture $(O W A)$ too small. 


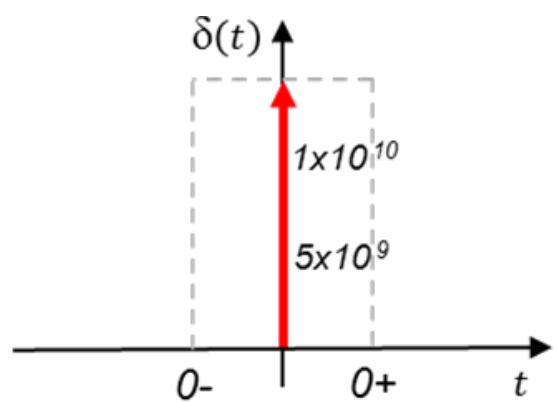

Fig. 5. The Delta of Dirac infinite pulse waveform with the DNAx approach.

\section{Modeling Some Important Digital Signals}

In the next two paragraphs, we intend to present two applications that we have been visualizing since this theoretical and numerical approach was introduced in the present literature some years ago. . $^{6,14}$

\subsection{New perspectives for modeling the pulse width modulation (PWM)}

At first, we will define the PWM sequence convergence process that can be modeled by the following analytical expression:

$$
f_{n}(t)=\left\{\left[\left(a_{p}(t)\right) \cdot X(t-d)^{n}+1\right]^{\left[1-\left(a_{p}(t)\right] \cdot X(t-d)^{n}\right]}\right\},
$$

where $a_{p}(t)$ is the modulation index, $X(t)$ is a reference signal designed with the PWM frequency, $d$ is the temporal shift, and $n$ is a positive integer number.

Also for this case, the PWM signal can be defined in the limit condition with the analytic representation in the form:

$$
f(t)=\operatorname{Lim}_{n \rightarrow \infty}\left[f_{n}(t)\right] .
$$

For this case, we define the index $a_{p}(t)$ as a real polynomial of the modulating signal $s(t)$. A very interesting analysis, which motivated our initials studies, is the case: $a_{p}(t)=$ constant, $X(t)=\sin (\omega \cdot t), d=0$. Figure 6 depicts the $2 \mathrm{D}$ graphics for the PWM signal $f(t)$ for the following choice of the parameters: $a=100$, and $\omega=\omega_{\text {pwm }}$.

\subsubsection{Relationship between the modulation index and the pulse width}

First of all, we suppose that with some additional information about the percent of the Duty Cycle (DC) it is possible to determine the dependence of index modulation $a_{p}(t)$ as a function of the modulating signal $s(t)$. Thus, in this case the pulse width can be defined by: $p_{d}(t)=2 /[y(t)]$, where $y(t)=\left(a_{p}(t)\right) \cdot\left\{|(d / d t)[X(t)]|, t \in\left[0, T_{m}\right]\right\}$, $T_{m}$ is the period of the PWM wave. ${ }^{19}$ 


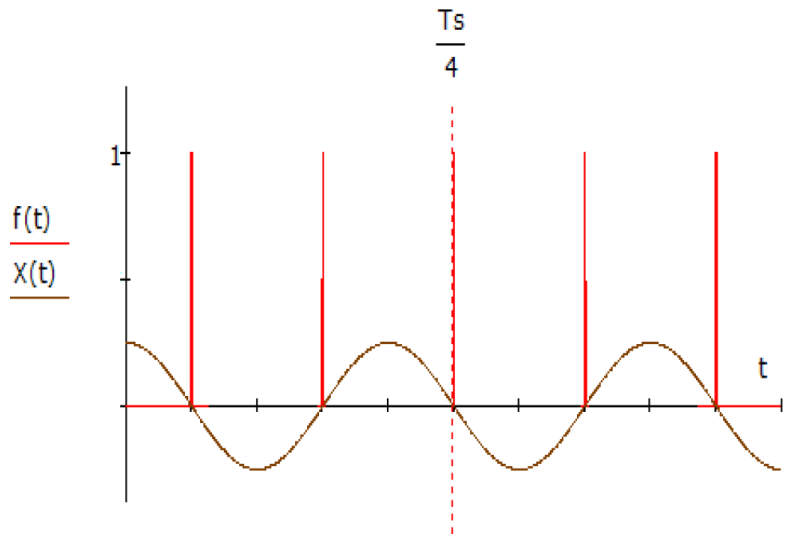

Fig. 6. A particular PWM signal $f(t)$ for the case: $a_{p}(t)=100, X(t)=\sin (\omega \cdot t)$ and $d=0$.

For this simple case, the reference signal $X(t)=\cos (\omega \cdot t)$ and the pulse width parameter will acquire the analytical form $p_{d}(t)=2 /\left[\omega \cdot\left(a_{p}(t)\right)\right]$. For an application, we must impose some requirements with respect to the modulated signal in accordance with the percent of the duty cycle.

\subsubsection{An application to be used for audio systems}

In our application, we assume $f_{m}$ is the PWM frequency and the audio signal $s(t)=2 \cdot\left[\sin \left(\omega_{s} \cdot t\right)+1\right], f_{s}=12(\mathrm{KHz})$. The reference signal $X(t)=\cos \left(\omega_{m} \cdot t\right)$, $f_{m}=60(\mathrm{KHz})$. For this case, it is possible to check that the PWM signal varies linearly, the values of the percent DC belong to the interval $[5,85](\%)$ and the modulating signal $s(t)$ has all values belonging to the interval [Min, Max].

Figure 7 shows the graphic for the 5.0(\%) DC, with all signals $s(t), X(t)$ and $f(t)$. For the DC percent we have the injective correspondence: $100(\%) \leftrightarrow\left(T_{p} / 2\right)$ and $5(\%) \leftrightarrow(0,05) \cdot\left(T_{p} / 2\right)$

In Table 1, we include all values of the percent of the DC (\% DC), the voltage amplitude of the signal $s(t)$ and the index $a_{p}(t)$, with the corresponding requirements imposed over the modulated output signal.

In Fig. 8, we present the relationship between PWM wave $f(t)$ and both normalized reference $X(t)$ and $s(t)$ signals, $n(t)=1000$ and $d=0$. In this non-forced case the best modulation index expression is: $a_{p}(t)=1.03+(7.2)(E-2) \cdot(s(t))^{3}+$ $\left.(1.88)(E-2) \cdot(s(t))^{3}+(3.59)(E-10)\right) \cdot(s(t)) .{ }^{12}$

In Fig. 9, we present the graphic of $s(t)$ and $a_{p}(t)$, where the values of $a_{p}\left[k \cdot\left(T_{s} / 4\right)\right]$ are: $9.553,1.468$ and 1.03; for $k=1,2,3$; respectively. Finally, Fig. 10 shows the forced case, with the same $s(t)$ and $a_{p}(t)$ used previously, where the bandwidth $w$ for a generic $T_{j}$ inside the real interval $\left[(5 / 8) \cdot\left(T_{s} / 8\right),(7 / 8) \cdot\left(T_{s} / 28\right)\right]$, has value equal to $w=T_{j}=(3 / 40) \cdot\left(T_{s}\right)$. 


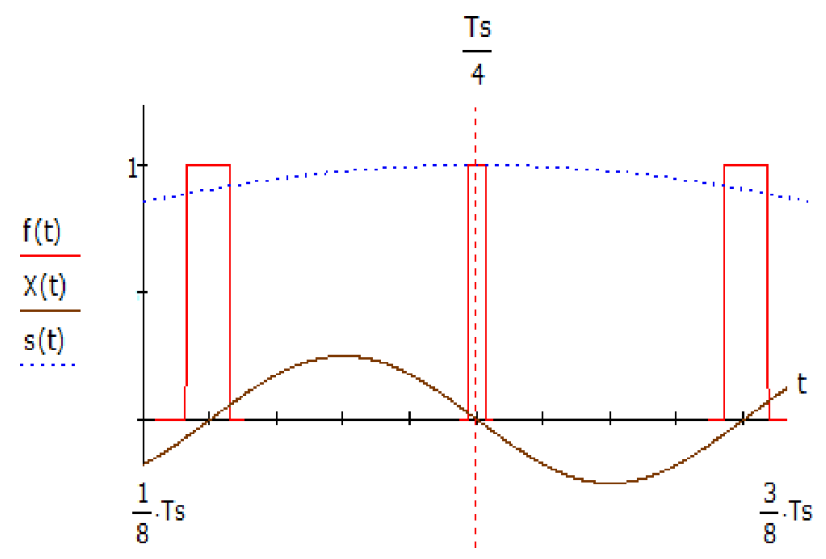

Fig. 7. The (5.0) $\%$ DC for the time $t=T_{s} / 4[s(t) \max ]$, with all signals $f(t), s(t) / 4$ and $X(t) / 4$.

Table 1. Modulation index and DC and normalized $s(t)$.

\begin{tabular}{ccccccc}
\hline Parameters $(\mathrm{P})$ norms $(\mathrm{N})$ & $(\mathrm{P})$ & $(\mathrm{N})$ & $(\mathrm{P})$ & $(\mathrm{N})$ & $(\mathrm{P})$ & $(\mathrm{N})$ \\
\hline$(\% \mathrm{DC})$ & 85.0 & $-\cdot-$ & 45.0 & $-\cdot-$ & 5.0 & $-\cdot-$ \\
$s(t)$ & 0.0 & 0.0 & 2.0 & 0.5 & 4.0 & 1.0 \\
$a_{p}(t)$ & 1.03 & $-\cdot-$ & 1.47 & $-\cdot-$ & 9.05 & $-\cdot-$ \\
\hline
\end{tabular}

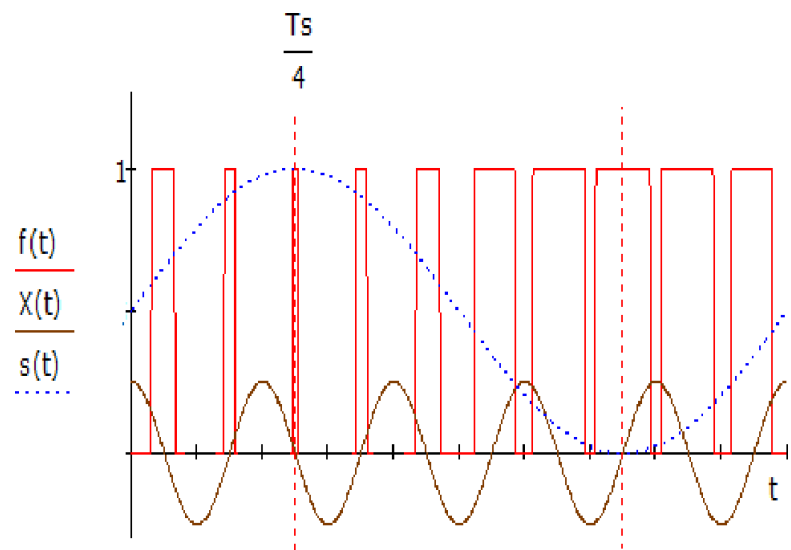

Fig. 8. Relationship between the $f(t), s(t)$ and $X(t)$ signals for the non-forced case.

\subsubsection{General analytical approach and the block diagram for the} implementation of the DNAx cells

For the more general approach, we will have a similar expression of (19), where the parameter $n=n(t)$, and the new process of convergence will start by defining 


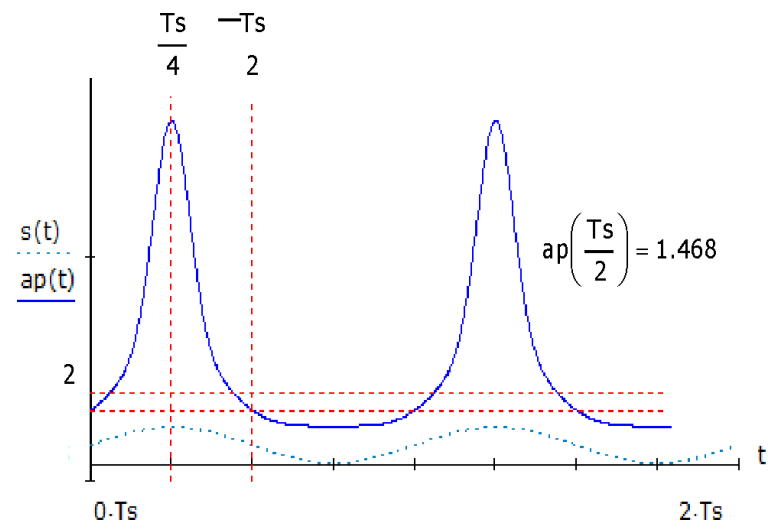

Fig. 9. Graphic of the normalized modulating signal $s(t)$ and the index one $a_{p}(t)$.

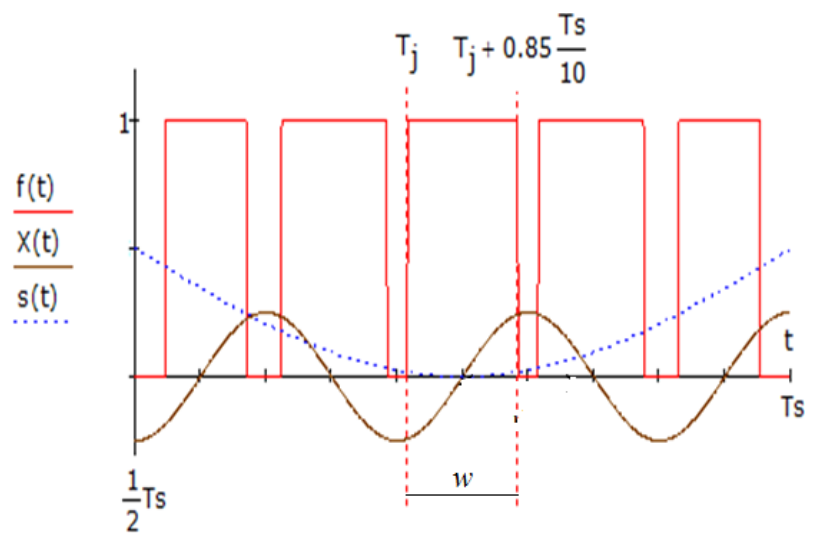

Fig. 10. The (85.0) \% DC for $t=3 \cdot T_{s} / 4[s(t) \mathrm{min}]$, with all signals $f(t) s(t) / 4$ and $X(t) / 4$.

the wave:

$$
f_{n}(t)=\left\{\left[\left(a_{p}(t)\right) \cdot X(t-d)^{n(t)}+1\right]^{\left[1-\left(a_{p}(t)\right) \cdot\left(X(t-d)^{n(t)}\right]\right.}\right\}
$$

and the new $P W M$ signal has the same definition for the limit condition performed in the previous analysis. In this case, we need to check that both parameters $n$ and $a_{p}$ are dependent on the time $t$.

In order to get some idea of how to implement the DNAx cells, in Fig. 11 we depict the ideal approach to establish the control of the system, where we present how to build the block diagram that permits us to obtain the cells for this particular model. Details of an architecture designed by using a chip of the Analog Device Co. can be found in others papers or theses published by the authors of Refs. 5, 7, 11 and 18. 


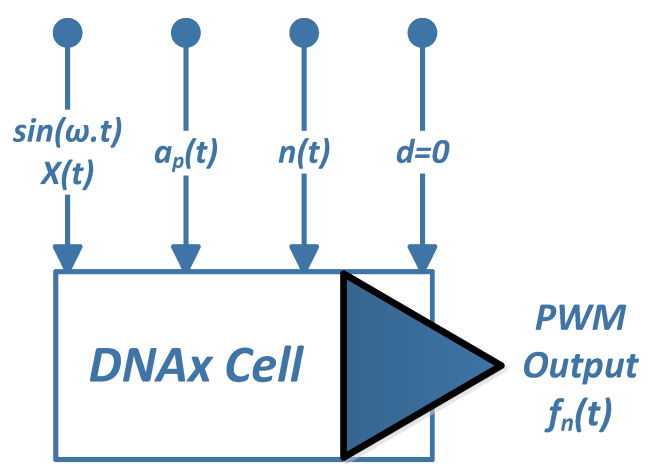

Fig. 11. Block design to implement the DNAx cell for the generation of the PWM stream.

It is easy to detect all essential parameters of the theoretical approach presented in this paper, and this procedure can be adopted for a wide class of audio systems. As an example, the PWM modulator can be considered as one DNAx cell with a dedicated equalization equation that can be readily programmed on a microchip.

\subsection{A new theoretical approach for digital modulation systems with a very fast Rise Time}

The essential purpose of this paragraph is to present a new theoretical concept for all digital systems that have rigorous requirements with respect to response time, which the authors will introduce as the Rise Time Modulation (RTM). The methodology adopted here is built on the foundation of the roll-off effect inserted in the theory of the Nyquist pulses, with direct application in the telecommunication industries. ${ }^{1,2,7,11}$

\subsubsection{Fundamentals properties for the DNAx operator}

At first we need to review an equivalent property for general pulses represented by the following equation:

$$
\left\{\left[\left[1+(a t)^{2 n}\right]^{\left[1-(a t)^{2 n}\right]}\right] f(t)\right\}=\left\{\left[(a t)^{2 n}+f(t)\right]^{\left[1-(a t)^{2 n}\right]}\right\}
$$

valid for an arbitrary $f:(-\propto,+\propto) \rightarrow \mathrm{R}$ continuous real function, $a>0, n \in N$ and the time $t \in[-a, a],[11],[1]$. A special application of Eq. (11) permits us to use a more appropriate formulation for a generic digital signal $f_{j}$ in the form:

$$
N_{j}\left[f_{j}(t)\right]=\left[(a \cdot t)^{2 n}+f_{j}(t)\right]^{\left[1-(a \cdot t)^{2 n}\right]} .
$$

6.2.2. Relationship between our theoretical approach and the classic Nyquist modeling for a digital pulse

Even though the theoretical analysis presented here can be applied for generic pulses modeled by the DNAx Eq. (22) if we make a proper manipulation over the 


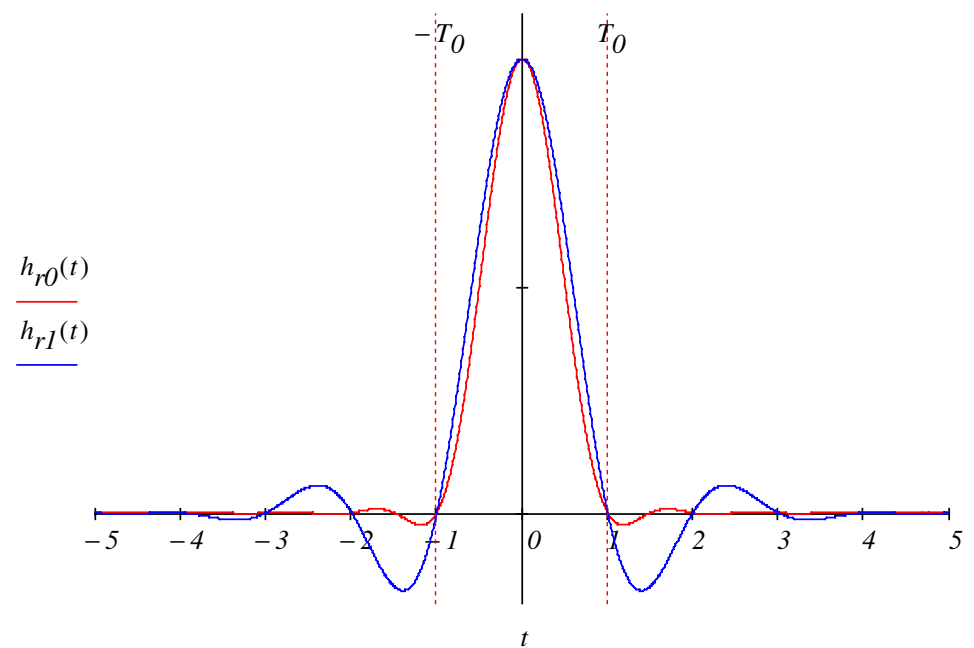

Fig. 12. Raised Cossine Nyquist pulses that are corresponding to the values: $r=1$ (Red), $r=0.35$ (Blue) and $t \in[-5,5]$ (color online).

parameters of the operator, it is easy to generate a Nyquist pulse with a desirable rolloff factor $r_{j}$.

Therefore, let us to consider the Raised Cosine function $h_{r}^{j}(t)$ defined by the equation:

$$
h_{r}^{j}(t)=\frac{(\sin c) \cdot\left[\pi \cdot\left(\frac{t}{T_{o}}\right)\right] \cos \left\{\left[\left(r_{j} \cdot \pi\right) \cdot\left(\frac{t}{T_{o}}\right)\right]\right\}}{\left\{1-\left[\left(2 \cdot r_{j}\right) \cdot\left(\frac{t}{T_{o}}\right)^{2}\right]\right\}}
$$

Figure 12 depicts two different pulses $h_{r}^{j}(t)$ corresponding to the values $r_{0}=1.0$ and $r_{1}=0.35$. For these cases, both analytical expressions are represented by the form:

$$
\begin{aligned}
h_{r}^{0}(t)=\frac{(\sin c) \cdot\left[\pi \cdot\left(\frac{t}{T_{o}}\right)\right] \cos \left[(\pi) \cdot\left(\frac{t}{T_{o}}\right)\right]}{\left\{1-\left[(2) \cdot\left(\frac{t}{T_{o}}\right)^{2}\right]\right\}}, \\
h_{r}^{1}(t)=\frac{(\sin c) \cdot\left[\pi \cdot\left(\frac{t}{T_{o}}\right)\right] \cos \left[(0.35) \cdot \pi \cdot\left(\frac{t}{T_{o}}\right)\right]}{\left\{1-\left[(2) \cdot(0.35)\left(\frac{t}{T_{o}}\right)^{2}\right]\right\}} .
\end{aligned}
$$

Figure 13 shows the superposition of the waves, with the same previous values of $r_{0}$ and $r_{1}$, and we emphasize the graphics of the function $h_{r}^{j}(t)$ for the more relevant interval; that is: $t \in[-1,1] ; j \in\{0,1\}$. 


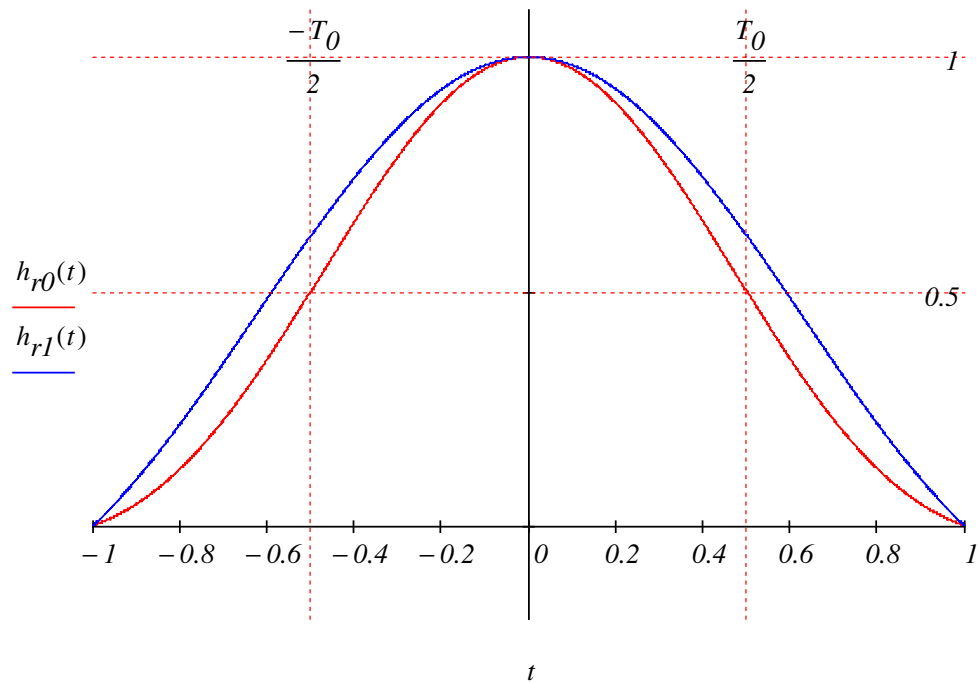

Fig. 13. Previous Raised Cossine Nyquist pulses that are corresponding to the values: $r=1$ (Red), $r=0.35$ (Blue) and $t \in[-1,1]$ (color online).

If we take into account the properties of the OWA introduced by the authors, by using a similar DNAx operator as in Eq. (22), we can to insert the Nyquist pulse inside a pre-determined time slot. ${ }^{3,4,6,7,18,20}$

In order to establish some relationship between the Nyquist pulses and the RTM approach, let us define the composite of the operators by the insertion of the Nyquist pulses in the generalized definition of the DNAx operator as is expressed in Eq. (22), which derives in the more simplified waveform defined by:

$$
N_{j}=\left[\left(N_{j}\right) \circ h_{r}^{j}\right](t)=\left[(a \cdot t)^{2 n}+h_{r}^{j}(t)\right]^{\left[1-(a \cdot t)^{2 n}\right]}, \quad j \in\{0,1\} .
$$

For the purpose of comparison with Fig. 13, let us to analyze the particular cases of the last operator, for the same functions $h_{r}^{j}(t)$ used in (24) and (25). In that case we have:

$$
N_{0}(t)=\left[(a \cdot t)^{2 n}+h_{r}^{o}(t)\right]^{\left[1-(a \cdot t)^{2 n}\right]}, \quad N_{1}(t)=\left[(a \cdot t)^{2 n}+h_{r}^{1}(t)\right]^{\left[1-(a \cdot t)^{2 n]}\right.} .
$$

It is easy to check if there is strong agreement between both $D N A x$ pulses $N_{j}(t)$ in the overlapping with the Nyquist pulses $h_{r}^{j}(t) ; j \in\{0,1\}$.

An important improvement of our approach, however, is the fact that we cannot to detect the presence of both undershoot and overshoot lobules with the exposition $D N A x$ pulses $N_{j}(t)$ just over the interval I $=[-1,1]$ and their vanishing outside of it. This does not occur with the original Nyquist pulses generated for the raised cosine in the same interval I. 


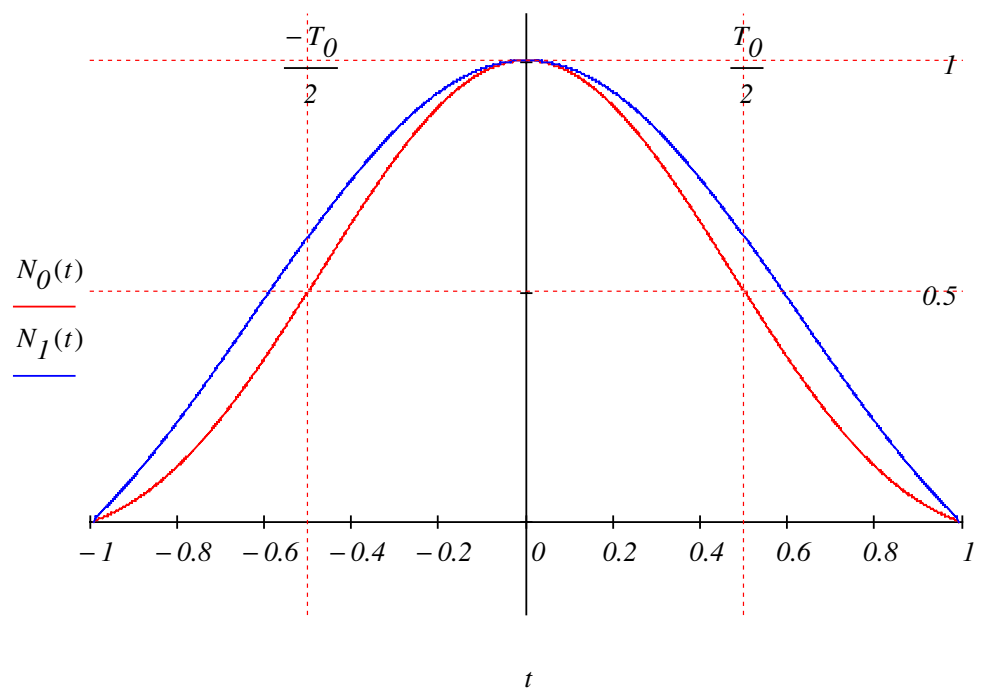

Fig. 14. Overlapping of the DNAx generalized pulse created as a composite with the Nyquist one.

Figure 14 shows the overlapping of the $D N A x$ generalized pulse created as a composite with the Nyquist one. For this case, in the Eq. (27) as above, we adopt the parameters: $a=\left(2 / T_{o}\right), n=10 \cdot(\operatorname{Exp}+06), j \in\{0,1\}$ and $t \in[-1,1]$.

In addition, it is easy to check that this analysis can be verified independently of any generic pulse we make as our reference signal to do some comparison. Furthermore, both $D N A x$ pulses $N_{j}(t)$ are the building blocks for the RTM modulation scheme; $j \in\{0,1\}$.

\subsubsection{Implementation of both Rise and Down Times}

In this paragraph, we intend to propose an efficient process to implement Rise and Down Times. With the procedure adopted in the previous paragraphs, it is possible to construct four new pulses which we can call Half pulses.

For $x_{o}(t)=(t+2)^{2 \cdot n}, x_{1}(t)=(t-2)^{2 \cdot n}, b_{n}(t)=\left[1-\left(x_{o}(t)\right)^{2 \cdot n}\right]$, let us define the pulses $p_{00}(t)$ and $p_{10}(t)$ by the equations:

$$
p_{00}(t)=\left[x_{0}(t)^{2 n}+h_{r}^{0}(t)\right]^{\left[b_{n}(t)\right]}, \quad p_{10}(t)=\left[x_{1}(t)^{2 n}+h_{r}^{0}(t)\right]^{\left[b_{n}(t)\right]} .
$$

In Fig. 15, we depict the 2D graphic of the signals $p_{00}(t)$ for the case: $a=0.5$ and $n=1000$. It is important to emphasize that the DNAx operator creates an OWA centered on the time $t=-T_{o} / 2$, with aperture equal to To. Inside of this time window, there is the composite of both DNAx and the Nyquist operators, which in this case implements the Rise Time.

It is important to check that outside the $O W A$ boundaries the operator $p_{00}(t)$ vanishes for all time scales. Applying a very similar procedure, we will be able to 


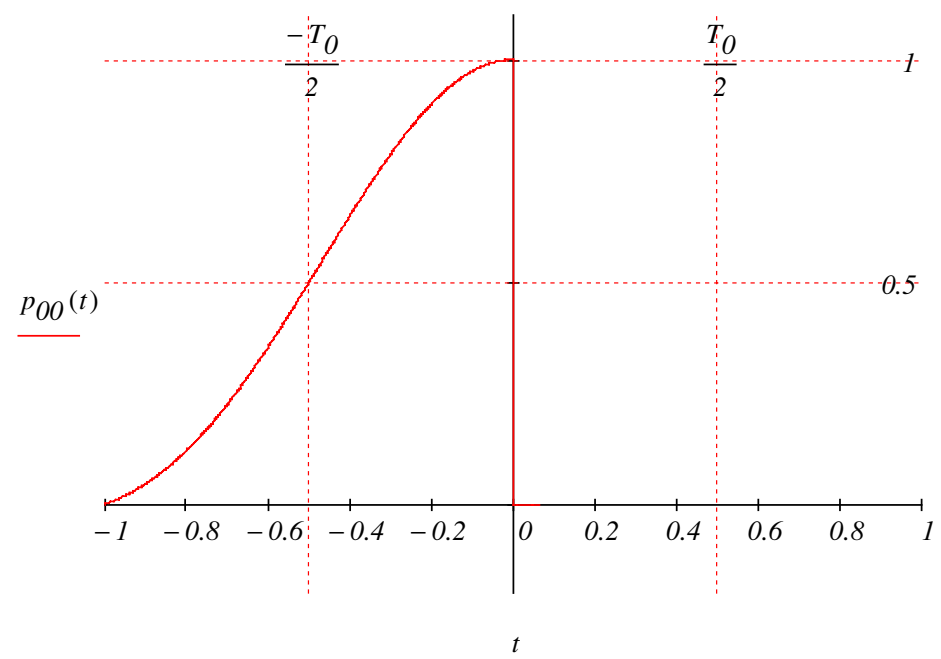

Fig. 15. 2D graphic for the Rise Time modulation signal built with the DNAx approach.

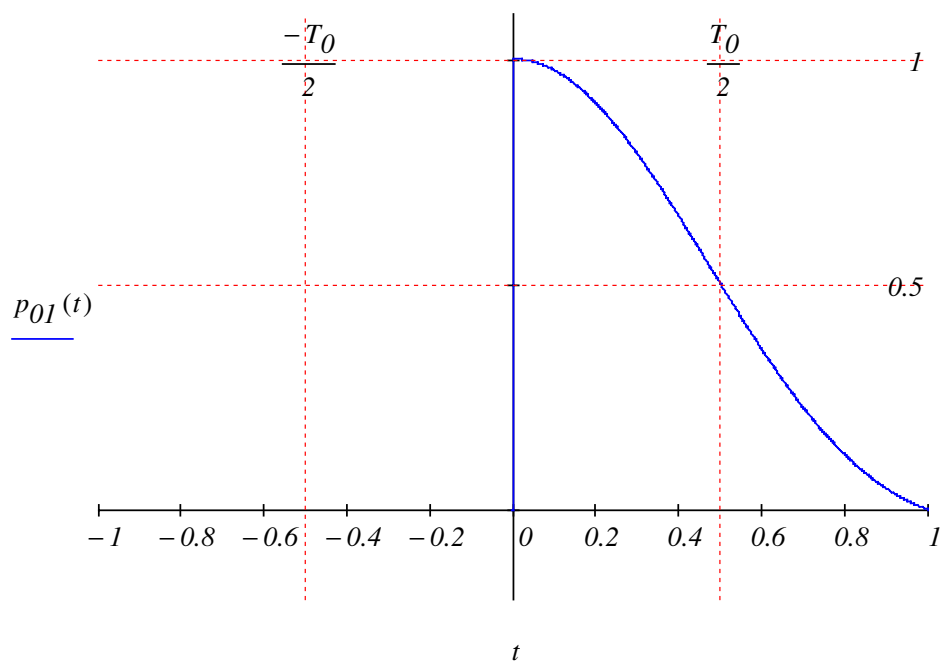

Fig. 16. 2D graphic for the Down Time modulation signal built with the DNAx approach.

Table 2. Values of the " $r$ " factor for both RTM modeling.

\begin{tabular}{lclll}
\hline Pulses & $P_{0}(t)$ & $P_{1}(t)$ & $P_{2}(t)$ & $P_{3}(t)$ \\
\hline Rise & 1 & 1 & 0.35 & 0.35 \\
Down & 1 & 0.35 & 1 & 0.35 \\
\hline
\end{tabular}


generate the $p_{01}(t)$ pulse waveform, as is shown in Fig. 16.

Since the $D N A x$ operator has a linear dependence as a function of the signal $f(t)$, we can add both previous pulses and create a new single signal $P_{0}$ in the form: $P_{0}(t)=p_{00}(t)+p_{10}(t)$, which permits us to recover the original Nyquist pulse as in Eq. (23).

Similar analysis can be done for the other combinations of the index "0" and " 1 "; $r_{1}=0.35$. Thus, it is possible to create the RTM modulation signal by varying the roll-off factor " $r$ " and in Table 2, we present all values for both RTMs.

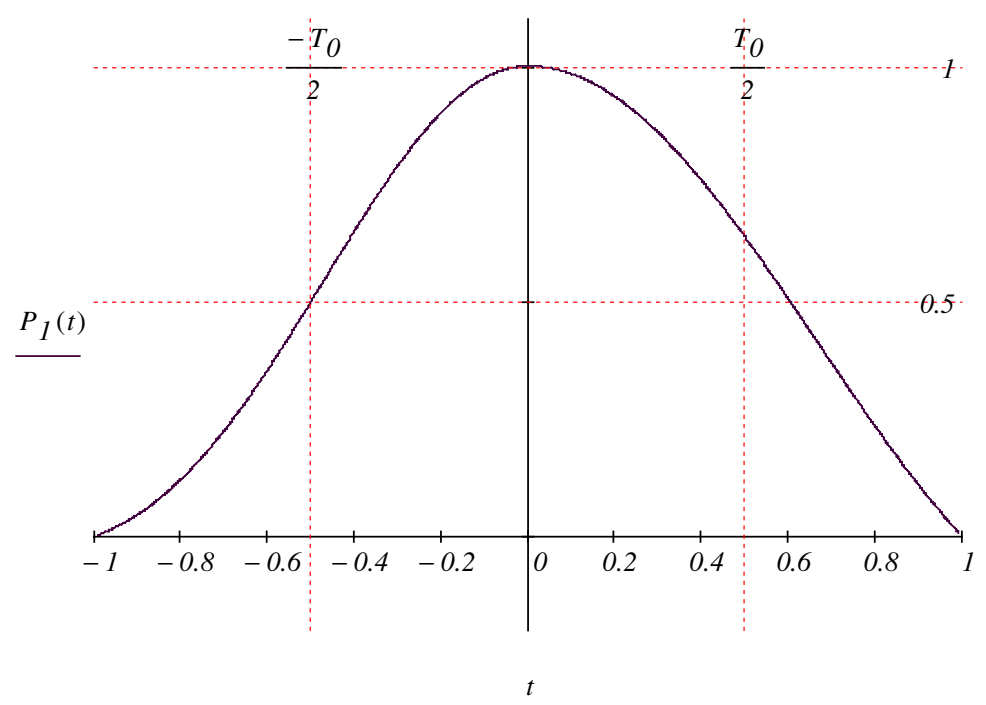

Fig. 17. Linear dependence between the DNAx functional operator and the Nyquist pulse approach.

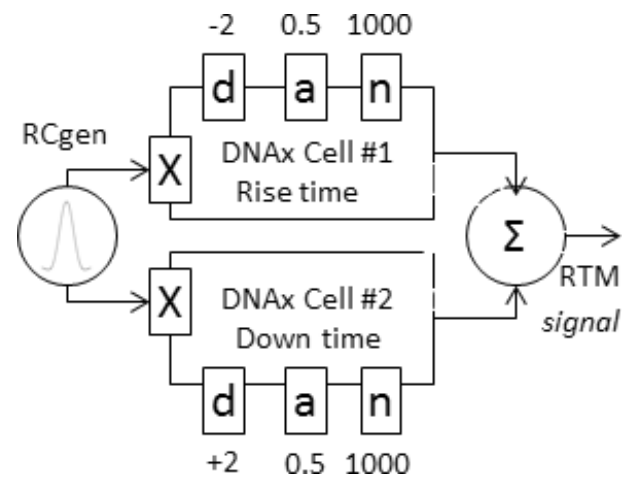

Fig. 18. Block diagram of an architecture used for setup the RTM pulses. 
In Fig. 17, we present the signal $P_{1}(t)=p_{00}(t)+p_{01}(t)$ and it is important to emphasize that both RTMs are plotted together depicted in the black color by adding the previous analytic representations of the signals $p_{00}(t)$ and $p_{01}(t)$, even though we still preserve the transmission of the Nyquist pulse.

Figure 18 depicts the diagram of an architecture that can setup the RTM pulses using essential parameters of the DNAx operator. . $^{5,11,18}$

\section{A Set of Future Lines of Research Involving this New Technology}

At this present moment, we are concentrating our main efforts on finding new directions where this theoretical as well as numerical formulation can be directly applied. The field of medical research could benefit greatly from this innovative technology, in the area of image vectorization, for instance there has already been some progress in this direction. ${ }^{16}$

Some advancement with this technology has been introduced for the first time in the current literature. We can to obtain an Analog signal as a finite sum of digital ones with a very fast numerical process of convergence. This generation of a digital rectangular waveform does not use any calculations involving integrals of real and periodic functions, as is formulated in the classic Fourier series approach. For that case, we need to determine both $a_{n}$ and $b_{n}$ as some real convolution without delay between some periodic Analog signal with the basis of AC signals $\boldsymbol{B}=\{\sin (n \omega \cdot t), \cos (n \omega \cdot t)\}$, which represents a set of countable harmonic periodic signals with distinct frequencies. In our process the generation of the classic rectangular waveform can be performed with a very easy process by the definition of this digital signal using the $D N A x$ operator. ${ }^{20,7}$

Furthermore, with the technological technique presented in this paper, it is possible to formulate a Reciprocal theorem of the Fourier series one that permits us to establish the generation of an Analog signal equal to the sum of a countable number of $D N A x$ pulses, by expressing a series of generic rectangular waveforms which are very similar to the one presented previously in Sec. 5.2. This is a great advancement in this original technological approach and we cannot detect in the current literature any other previous results similar to that one formulated by the Fourier approach. ${ }^{8,9}$

At this moment, there are not many areas of research related to digital communication where we have been able to apply this technological approach. A complete set of traditional Digital Pulses Streams, like for example: TDM, PWM, PPM, UWB, etc., can be easily modeled using this innovative technique, which is part of our continuous work in progress. ${ }^{15,17-19,23,24}$

In addition, with two recent applications of the $D N A x$ operator, some progress has been made with respect to the UWB technologies. We can also model the classic mother wavelets for the generation of the $2 \mathrm{D}$ plots of the electrocardiogram (ECG) that has been used widely in the field of medical research. ${ }^{12,13}$ 


\section{Conclusions}

The approach presented in this paper permits us to obtain a new technological tool that can be used for modeling analytically a wide class of important electromagnetic signals and waveforms by using a simple and efficient theoretical as well as numerical technique.

The purpose of this paper is to introduce to the scientific community the $D N A x$ technique illustrated with the Slew Rate modulation. Now, besides the phase modulation, amplitude modulation, and frequency modulation, another parameter is made available for modulation. This new parameter is totally in accordance with the Nyquist bandwidth criteria. ${ }^{10,11}$

Many other electromagnetic effects can be analyzed with this theoretical approach, and one of the most important is concerned with a new formulation of a theorem similar to the classic Fourier series approximation. ${ }^{8,9}$ For many years this was the unique main prop in the digital communication area which provided engineers with the fundamental principles involving the projects of the $D / A$ electronic converters. ${ }^{14}$

We hope these analytical definitions introduced for the first time into the literature reflect our modest contribution to the development of new technologies in the area of research for digital communication systems. At the present moment, we are concentrating our efforts on finding new applications to enhance the interaction between the fields of engineering and medicine. ${ }^{13,21,22}$ This research is strongly supported by Academic institutions as well as by many Institutes of research. ${ }^{15-17}$

\section{Acknowledgments}

Our thankfulness for the Perfect Pulse Corporation, Inc., Geneva, IL, USA, which gave us permission to publish this research and the São Paulo Research Foundation, FAPESP, SP, Brazil, for partial financial support received by the co-author of this paper under processes 2006/01655-7 and 2013/23431-7.

\section{References}

1. A. V. Oppenheim and R. W. Schaffer, Discrete Signal Processing, 3rd edn. (Prentice Hall, 2009).

2. R. G. Lyons, Understanding Digital Signal Processing Communications, Fundamental and Applications, 3rd edn. (Prentice Hall, 2010).

3. R. C. Dorf and R. H. Bishop, Modern Control Systems, 12th edn. (Prentice Hall, 2010).

4. E. M. Stein and R. Shakarchi, Functional Analysis, Introduction to Further Topics in Analysis (Princeton University Press, 2011).

5. R. G. Caputo, Um Novo Método de Geração de Sinais Compostos, MSc. Degree Thesis, National Institute of Telecommunication, INATEL, Minas Gerais, Brasil, February 2006.

6. S. Çikek, Y. Uyaroglu and I. Pehlivan, Simulation and circuit implementation of sprott case $\mathrm{H}$ chaotic system and its synchronization application for secure communication systems, J. Circuits, Syst. Comput. 22 (2013) doi:10.1142/S0218126613500229. 
7. R. G. Caputo and G. V. Figueiredo, Method of generating complex waveforms and modulating signals. USA Provisional Patent Application Serial: Nr. 60/543,191, 2003; Patent: Nr.10/463, 482, 2004 and Patent: Nr.7/801, 936, 2010.

8. R. G. Caputo, G. V. Figueiredo and M. Silveira, An innovative theoretical contribution for the Direct and Reciprocal Fourier numerical convergence process to generate of a wide class of Analog and Digital Signals, IEEE DINWC, Ostrava, Czech Republic, 2014, unpublished.

9. R. G. Caputo, G. V. Figueiredo and M. Silveira, An efficient and innovative approach for the direct and reciprocal Fourier numerical with a very high speed for the generation of a wide class of Analog and Digital Signals, Int. J. Commun. Syst. IJCS, Malden, MA, USA, unpublished.

10. R. G. Caputo, G. V. Figueiredo and M. Silveira, A new theoretical modeling for the Nyquist pulses processing used widely over communication channels for systems with high transmission rates, IEEE ICIA, Lodz, Poland, 2013, doi: 10.1109/ICoIA.2013.6650251.

11. R. G. Caputo, G. V. Figueiredo and M. Silveira, A new theoretical approach for digital modulation used widely for systems with a very Fast Rise Time, IEEE ICIA, Lodz, Poland, 2013, doi: 10.1109/ICoIA.2013.6650250.

12. C. Almeida, Synthesis of the UWB Pulses using the DNAx approach, MSc. Degree Thesis, National Institute of Telecommunication, INATEL, Minas Gerais, Brazil, March 2009.

13. M. C. Moraes, Analysis of ECG Signals using Wavelet Transform with the DNAx Operator, MSc. Degree Thesis, National Institute of Telecommunication, INATEL, Minas Gerais, Brazil, May 2008.

14. R. G. Caputo, G. V. Figueiredo and M. Silveira, Innovative theoretical modeling for the pulse point level detection on A/D converters involving Pulse Position Modulation, IEEE ICIA, Lodz, Poland, 2013, doi: 10.1109/ ICoIA.2013.6650252.

15. R. G. Caputo, G. V. Figueiredo and M. Silveira, An innovative numerical technique with high speed time processing for localizes the zeros of a wide class of real polynomials. Direct Applications for systems that demand the Digital Pulse Position Modulation, IEEE ICIA, Lodz, Poland, 2013, doi: 10.1109/ ICoIA.2013.6650.

16. R. G. Caputo, G. V. Figueiredo and M. Silveira, Implementing an innovative theoretical proposal with optimization in the time of computational processing involving Image Vectorization techniques, IEEE ICIA, Lodz, Poland, 2013, doi: 10.1109/ICoIA.2013.6650254.

17. R. G. Caputo, G. V. Figueiredo and M. Silveira, A new efficient approach for modeling the Ultra Wide Band Systems. Applications for links involving wireless digital communications, IEEE $R W W$, Newport Beach, CA, USA, 2014, unpublished.

18. R. G. Caputo, G. V. Figueiredo and M. Silveira, Checking the compatibility between Original analytical and experimental electromagnetic waveforms, IEEE APS/URSI, Albuquerque, NM, USA, 2006.

19. R. G. Caputo, G. V. Figueiredo and M. Silveira, New perspectives for modeling the Pulse Width Modulation. Some application for audio systems, IEEE ICIA, Lodz, Poland, 2013, doi: 10.1109/ICoIA.2013.6650247.

20. R. G. Caputo, G. V. Figueiredo and M. Silveira, A new analytical accurate analysis to generate classical communication signals, IEEE APS/URSI, Albuquerque, NM, USA, 2006.

21. G. Ferri, V. Stornelli and A. Simone, A CCII-based high impedance input stage for biomedical applications, J. Circuits, Syst. Comput. 20 (2011) doi:10.1142/S021812661100802X.

22. R. A. Khalid and R. Paily, FPGA implementation of high speed and low power architectures for image segmentation application using sobel operators, J. Circuits, Syst. Comput. 21 (2012) doi:10.1142/S0218126612500508. 
23. Caputo, R.G., Figueiredo, G.V., Silveira, M., An accurate theoretical approach for the generation of Finite Digital Pulses. An application for modern Electronic Digital Architectures, IEEE DINWC 2014, Ostrava, Czech Republic, unpublished.

24. Caputo, R.G., Figueiredo, G.V., Silveira, M., Data Comprehension Code for Fiber Optics Application, Demo Track Proposal, Invited Talking, IEEE RWW 2014, New Port Beach, CA, USA, unpublished. 